\title{
Adaptación social en jóvenes judicializados que participan en actividades escolares
}

\section{Social Adjustment in Prosecuted Young Offenders Involved in School Activities}

\author{
Leticia Rita Lorenzino ${ }^{\mathrm{a}}$, Laura Inés Rivera-Betancourt ${ }^{\mathrm{a}}$, \& Griselda Cardozo ${ }^{\mathrm{a}, \mathrm{b}}$ \\ ${ }^{a}$ Universidad Nacional de Córdoba, Córdoba, Argentina \\ ${ }^{b}$ Universidad Católica de Córdoba, Córdoba, Argentina
}

\begin{abstract}
Resumen: El propósito de esta investigación fue analizar la adaptación social (conductas prosociales y antisociales) de 86 jóvenes infractores de la ley de entre 13 y 21 años, que asisten a la escuela de un instituto socioeducativo de puertas cerradas en la ciudad de Córdoba, Argentina. Se utilizó un diseño de tipo descriptivo correlacional. Los resultados demuestran que los jóvenes presentan -en relación con las variables que miden conducta prosocialbajos niveles de empatía y respeto, altos niveles de sociabilidad y liderazgo. En relación con la conducta antisocial, los niveles alcanzados para agresividad, retraimiento y aislamiento son altos. En lo que respecta al clima prosocial escolar el nivel percibido por los alumnos es bajo. El análisis correlacional indica relaciones significativas entre la conducta prosocial de los jóvenes, la percepción que tienen acerca de la conducta prosocial de sus pares en el aula y el clima prosocial escolar. Es decir, quienes perciben un clima prosocial escolar más alto, suelen ser más prosociales y viceversa. Por lo cual se considera que la implementación de programas educativos que promuevan una mejora en el clima prosocial del aula podría contribuir al fortalecimiento de la conducta prosocial a nivel individual de los jóvenes que asisten a la escuela, logrando una mejor adaptación social.
\end{abstract}

Palabras clave: adaptación social, jóvenes, delincuencia, escuela.
Abstract: The aim of this research was to analyze the social adjustment (prosocial and antisocial behaviors) of 86 young offenders -between 13 and 21 years of age- attending a correctional school of a socioeducative institute in Córdoba, Argentina. A descriptive/correlational design was used for the analysis. The results show that young offenders have -with regard to the variables measuring prosocial behavior- low levels of empathy and respect and high levels of sociability and leadership. What concerns antisocial behavior, the levels reached for aggression, withdrawal and isolation are high. On the other hand, the collective perception in school of a prosocial atmosphere is low. The correlational analysis indicates significant relationships between prosocial behavior of young offenders, their perception of prosocial behavior among peers in the classroom, and the school prosocial atmosphere. This means that young offenders, who perceive a higher prosocial atmosphere in school, should be more prosocial and vice versa. Therefore, it is considered that the implementation of educational programs design to promote an improvement in the prosocial atmosphere of the classroom, could also contribute to strengthen prosocial behavior in young offenders attending school, thus achieving a better adjustment to their social environment.

Keywords: social adjustment, young offenders, delinquency, school.

La presente investigación forma parte del proyecto de investigación "El rol de la escuela en la formación de jóvenes resilientes: implementación de un programa educativo en habilidades sociales" financiado por la Secretaría de Ciencia y Tecnología (Secyt) de la Universidad Nacional de Córdoba (UNC). Este trabajo ha sido posible gracias al apoyo de la Secretaría de Niñez, Adolescencia y Familia (Senaf), de la ciudad de Córdoba, Argentina.

Contacto: L. R. Lorenzino. Obispo Salguero 557 - 9 B, Córdoba, Argentina. CP: 5000. Correo electrónico: leticialorenzino@gmail.com

Cómo citar: Lorenzino, L. R., Rivera-Betancourt, L. I., \& Cardozo, G. (2015). Adaptación social en jóvenes judicializados que participan en actividades escolares. Revista de Psicología, 24(2), 1-18.

http://dx.doi.org/10.5354/0719-0581.2015.37507 


\section{Introducción}

La teoría del aprendizaje social de Bandura (1977) ha sido retomada por los estudios criminológicos para explicar el comportamiento delictivo. Actualmente los trabajos de Ronald Akers se consideran los más aceptados en las investigaciones en este ámbito, quien retoma a su vez, la teoría de la asociación diferencial de Sutherland (Akers, 2009; Graña Gómez \& Rodríguez Biezma, 2010; Redondo Illescas, Martínez Catena, \& Andrés Pueyo, 2011). Estas teorías que explican la delincuencia, si bien están enfocadas en observar la conducta de las personas, también sostienen que se debe hacer una lectura de las variables cognitivas que forman parte de los mecanismos básicos del aprendizaje (Akers \& Burgess, 1966).

Según Farrington (1998) se puede considerar delincuente juvenil a toda persona que haya cometido un delito y que tenga entre 10 y 21 años. Afirma que aquellos adolescentes que han cometido alguna vez un delito tienden a seguir cometiéndolos y eso, a su vez, se correlaciona con actividades como las de abuso de sustancias o relaciones sexuales prematuras. También se debe tener en cuenta que en este rango etario es donde más se presenta este tipo de conductas antisociales y que luego muchas de ellas desisten en el tiempo. Según Moffitt (1993) alrededor de los 20 años disminuye en un $50 \%$ la población de jóvenes delincuentes y a los 28 años el $85 \%$ deja de delinquir, pero queda un pequeño porcentaje de jóvenes que persiste en el tiempo, agravando su conducta.

Por lo general, los jóvenes delincuentes no tienen una carrera delictiva específica, sino que tienden a llevar a cabo diferentes tipos de crímenes y a exhibir otros problemas como deserción escolar, abusos de sustancias, mentiras y relaciones sexuales pro- miscuas; sin embargo, se entiende que hay un pequeño grado de especialización impuesta a su versatilidad (Farrington, 1998; Moffit, 1993; Moffit, Caspi, Harrington, \& Milne, 2002).

Farrington (1998) estudia aquellas características particulares que se presentan según la edad y tienden a ser predictoras de la posible delincuencia juvenil de los sujetos, arribando a la conclusión de que entre $\operatorname{los} 8$ y $\operatorname{los} 10$ años estos niños se caracterizan por tener dificultad en la disciplina, ser problemáticos y poco honestos; entre los 12 y los 14 años tienden a ser agresivos, cometen bullying, mienten de manera frecuente, reaccionan hostilmente frente a la policía, tienen una pandilla con amigos delincuentes, dejan la escuela de manera temprana y tienen relaciones sexuales prematuras; por último, alrededor de los 18 años se observa que estos sujetos muestran violencia en el fútbol, participan en grupos antisociales, consumen drogas, cigarro y alcohol, manejan en estado de ebriedad, evidencian una actitud agresiva y poco estable, sus trabajos son poco constantes y no bien remunerados y muestran malas relaciones con los padres.

Según Moffitt $(1993,2005)$ resulta necesario diferenciar a aquellos jóvenes que tienen una carrera delictiva esporádica y no continua, la cual se debe a los roles de la etapa adolescente (que por lo general desaparecen con la edad), de aquellos que tienen una carrera delictiva persistente expuestos a múltiples factores de riesgo. Son estos últimos los que necesitan más atención, debido a que tienen también comportamientos antisociales marcados en la temprana infancia.

De acuerdo con lo desarrollado por Farrington (1998) se observa que los mayores predictores o factores de riesgo de la delincuencia juvenil suelen ser aquellos relacio- 
nados con lo biológico, individual, familiar $\mathrm{y}$ ambiental, en tanto exista un bajo nivel socioeconómico, una escuela vulnerable y un lugar de residencia en un vecindario conflictivo. A su vez, Redondo Illescas et al. (2011) sostienen que el hecho de que un joven delincuente se convierta en un adulto delincuente, se relaciona con el grado de motivación antisocial que tenga a partir de los factores de riesgo que se presenten.

Sin embargo, no toda persona que se encuentra sometida a factores de riesgo desarrolla necesariamente comportamientos de riesgo, lo cual se debe a que una gran proporción de la población vulnerable que se encuentra expuesta a estos aspectos cuenta con factores protectores. El factor de protección es entendido como un elemento que disminuye el riesgo de presentar comportamientos inadaptados por parte de aquellos individuos que se encuentran expuestos a factores de riesgo en su medio ambiente (Trudel, Puentes Neuman, \& Ntebutse, 2002).

Desde una perspectiva conductual el comportamiento prosocial se contempla como un gran componente en la extinción de la agresividad o violencia, dado que se considera una respuesta incompatible con estas. Roche Olivar propone que la introducción, ejercicio y aumento en la cantidad y calidad de actitudes y comportamientos prosociales constituye una vía simple y segura de educación emocional orientada hacia el bienestar y salud mental de la persona, como así también una mejora en su convivencia social (Cirera Amores, Escotorín Soza, \& Roche Olivar, 2008).

Las conductas prosociales o antisociales son resultantes de la internalización de esquemas referenciales cognitivoemocionales, que promueven la actuación en relación con un determinado sistema de valores y creencias. Por lo tanto, para po- der comprender la importancia de la conducta prosocial es indispensable retomar conceptos que se aproximen a nociones de socialización, contemplando modos de adquisición y desarrollo de dichas conductas, como así también visualizar el rol de los agentes socializadores como la familia y la escuela, en la transmisión de valores, normas y reglas (González, Casullo, Martorell, \& Calvo, 1998).

De acuerdo con lo postulado por Alarcón Bañares et al. (2010) la adaptación social es un proceso evolutivo donde los comportamientos pueden desviarse de las normas sociales, transgrediendo reglas o límites establecidos por la cultura. Martorell, González, Ordoñez, \& Gómez (2011b) señalan que en la adolescencia la empatía, el respeto por los demás, las relaciones sociales y el liderazgo se encuentran en evolución, manteniendo una relación inversa con variables como la impulsividad y las conductas antisociales. Por lo tanto, la juventud es una etapa de transición en donde se consolidan habilidades interpersonales futuras del individuo, de allí la importancia de promover un correcto desarrollo de este tipo de conductas.

Para Casullo (1998) la adaptación social está compuesta por las conductas prosociales y antisociales. Martorell et al. (2011b) definen la conducta prosocial como "conducta social positiva" (p. 35) e implica conductas de ayuda, cooperación, intercambio y cumplimiento de normas. Atendiendo a estas dimensiones, la empatía es definida como "la capacidad de ponerse en el lugar del otro e intentar aliviar su malestar" ( $p$. 42); en tanto que la sociabilidad se entiende como la "capacidad de llevar a cabo relaciones sociales positivas" (p. 43); el respeto, por su parte, se concibe como la "capacidad para tratar a los demás con asertividad" (p. 43) y por último el liderazgo se entiende como la "capacidad de organizar y 
dirigir actividades en grupo" (p. 43). Por su parte, la conducta antisocial es definida como "todo comportamiento que quebranta normas e intereses sociales, siendo una acción dañina para los demás" (Martorell, González, Ordóñez, \& Gómez, 2011a, p. 97). Atendiendo a las dimensiones, la agresividad comprende "toda conducta que implique agresividad verbal o física en relación con los demás" (p. 104); el retraimiento/ansiedad se han definido como "la dificultad para relacionarse con los demás, remarcando no tanto en los sentimientos de tipo psicológico, sino en las reacciones fisiológicas" (pp. 104-105) y el aislamiento como la "necesidad no adaptativa de estar solo, huyendo de situaciones que impliquen relacionarse con los demás" (p. 104).

A partir de la interacción con el ambiente, el niño va asimilando valores sociales producto de una elaboración constructiva personal que le permite regular su conducta. La socialización, supone, por lo tanto, la asimilación de normas, valores y reglas que pretenden regular la conducta a fin de lograr una adaptación a la sociedad. La educación de sentimientos de compasión, empatía y valores sociomorales es sumamente importante, ya sea desde la familia o la escuela, para desarrollar conductas prosociales en cualquiera de sus manifestaciones (Maganto, 1994).

La prosocialidad es un concepto que surge en psicología como antónimo del concepto antisocial, el cual generalmente es usado como adjetivo; sin embargo Roche Olivar (2011) ha empezado a utilizar el sustantivo prosocialidad como un modelo de pensamiento. El autor sostiene que uno de los modos más eficaces para la inhibición de conductas violentas y agresivas constituye la exposición del individuo a repertorios de conductas positivas y prosociales que sean funcionales en la consecución de objetivos personales y sociales.
De acuerdo con lo propuesto por Roche Olivar, la prosocialidad puede entenderse como:

Aquellos comportamientos que, sin la búsqueda de recompensas externas, favorecen a otras personas, grupos o metas sociales y aumentan la probabilidad de generar reciprocidad positiva, de calidad y solidaria en las relaciones interpersonales o sociales consecuentes, salvaguardando la identidad, creatividad e iniciativa de las personas o grupos implicados (...), conjuntamente arriesga una definición operativa de la prosocialidad caracterizándola como aquellas acciones que tienden a beneficiar a otras personas, sin que exista la previsión de una recompensa (Roche Olivar, 2011, p. 16).

Además, el autor postula categorías de la conducta prosocial, entre ellas menciona las siguientes: ayuda física (procurar asistencia física a otras personas para cumplir un determinado objetivo), servicio físico (implica intervenir en el cumplimiento de una tarea que corresponde a otro), dar (entregar objetos o posesiones), ayuda verbal (explicación verbal que es útil y deseable para otras personas o grupos en la consecución de objetivos), consuelo verbal (expresiones verbales para reducir la tristeza de otros, aumentando su ánimo), confirmación y valorización positiva del otro (expresiones verbales para confirmar el valor de otro o aumentar su autoestima incluso ante terceros), escucha profunda (conductas metaverbales y actitudes que expresan acogida interesada en los contenidos del interlocutor), empatía (conductas verbales que expresan comprensión cognitiva de los pensamientos o emociones del otro, experimentando sentimientos similares a los de este), solidaridad (conductas que expresan aceptación vo- 
luntaria de compartir consecuencias, especialmente penosas, de la condición o situación desgraciada de una persona, grupo o país) $\mathrm{y}$, finalmente, presencia positiva y unidad (actitud de proximidad psicológica, atención, escucha profunda, disponibilidad para la ayuda y solidaridad para con otras personas, contribuyendo a un clima psicológico de bienestar, reciprocidad y unidad) (Roche Olivar, 2011).

Por su parte, Barrón y Carbonetti (2006) mencionan que la posibilidad de acceder a la escolarización favorece cambios en el desarrollo cognitivo que propician la toma de decisiones adecuadas y la capacidad de prever las consecuencias de sus conductas a corto y largo plazo. A su vez, Lorenzo Moledo, Aroca Montolío y Alba Robles (2013) mencionan que la educación se transforma en un elemento fundamental de intervención en el ámbito penitenciario, no solo como parte esencial del tratamiento, sino por la consideración del interno como un sujeto de derecho que no está excluido de la sociedad.

En lo que refiere a estudios locales enfocados en la relación entre escuela y violencia resulta importante destacar el trabajo de Kornblit, Adaszko y Di Leo (2008) quienes definen al clima social escolar como "las percepciones que tienen los sujetos acerca de las relaciones interpersonales que establecen en el contexto escolar y el contexto o marco en el cual estas interacciones se dan" (p. 59). Mencionan que mientras el clima social escolar favorable puede disminuir las conductas violentas, dicha influencia no es absoluta y su límite se encuentra en relación con variables del entorno; sin embargo, a partir de los estudios realizados, concluyen que a medida que empeora el clima social escolar, se incrementan los episodios de violencia.
Romersi, Martínez Fernández y Roche Olivar (2011) en un estudio evalúan el clima prosocial percibido en el aula, el cual se vincula con la percepción de acciones positivas en general, destacando que los factores, ayuda física y la conducta inclusiva, parecen ser los de mayor impacto en la conducta prosocial individual y la percepción del clima colectivo en el aula. Igualmente, mencionan que el clima prosocial de la clase se aprecia en la importancia de los elementos de confirmación y valorización positiva del otro.

Por otro lado Crabay (2007) refiere que los comportamientos prosociales constituyen una herramienta eficaz en los procesos de aprendizaje e interacción, ya sea en instancias educativas formales como no formales. En esta línea, Roche Olivar (2011) menciona que uno de los factores que influye en el desarrollo de las orientaciones prosociales es el aprendizaje, haciendo énfasis en la imitación. De igual modo, destaca que en la aproximación al estudio de los comportamientos prosociales se pensó el trabajo desde la intervención educativa, por ser considerado el campo más idóneo para contribuir a una transformación a largo plazo. Señala que en la optimización de las conductas prosociales, un modelo colectivo prosocial puede ser el contexto educativo, enfatizando el rol que juega la identificación en todo proceso formativo. Así, Eberly y Roche Olivar (2002) destacan algunos aspectos del educador que son útiles en la enseñanza de la prosocialidad; entre ellos refieren la manifestación de afecto por los alumnos, la expresión de confianza y el refuerzo de actitudes prosociales a través de elogios.

Respecto de las intervenciones, Roche Olivar (2011) propone el modelo teórico práctico Unidades Prosociales (Unipro) como un marco para la creación y diseño 
de programas de educación de la prosocialidad, el cual trata de optimizar actitudes y comportamientos de ayuda, cooperación, generosidad, solidaridad, amistad y unidad. El programa se basa en una visión globalizadora y compleja del comportamiento humano, y está pensado para trabajar mediante sesiones y actividades de sensibilización cognitiva.

Finalmente, resulta importante destacar que la implementación de programas de incremento prosocial en la institución penitenciaria ha demostrado resultados significativos en la reducción de reincidencia. Entre ellos, actualmente en España el Programa de Pensamiento Prosocial es uno de los más utilizados con delincuentes juveniles, el cual apunta a trabajar con factores de riesgo cognitivos, emocionales y de conducta, demostrando su eficacia en el incremento de habilidades de interacción y disminución de sus distorsiones cognitivas y justificación del delito (Redondo Illescas, Martínez Catena, \& Andrés Pueyo, 2012).

Sobre la base de lo expuesto anteriormente, este estudio se propone como objetivo describir si existe relación entre el clima prosocial escolar y la adaptación social de los jóvenes infractores de ley que asisten a la escuela de un centro socioeducativo de la ciudad de Córdoba, Argentina, entre los meses de junio y noviembre del año 2014. El estudio tiene como finalidad arrojar luz en torno a la hipótesis de que existe una relación entre el clima prosocial escolar y el nivel de adaptación social de los jóvenes judicializados que asisten a la escuela.

\section{Método}

\section{Población}

Si bien la población total del centro socioeducativo está constituida por 250 jóvenes varones, de entre 10 y 21 años de edad que están en conflicto con la ley penal, para la realización del estudio - por diversos motivos institucionalesse seleccionó a los sujetos de modo no paramétrico accidental; quedando la muestra conformada por 86 jóvenes que participaban de las diferentes modalidades educativas que ofrece el instituto. Las edades de estos estudiantes variaron entre $\operatorname{los} 13$ y 21 años $(M=16,9 ; D E=$ $1,37)$.

La institución aloja a jóvenes hasta los 18 años; luego de haber cumplido esa edad deben culminar su condena en un centro penitenciario para adultos. Cabe aclarar que, en este caso, la franja etaria resulta tan amplia dado que en algunas situaciones se prioriza la integridad del joven $\mathrm{y}$, si es necesario, culmina su condena en el instituto para menores.

Los jóvenes que asisten a la escuela en el centro socioeducativo son distribuidos en las distintas modalidades de formación de acuerdo con el instituto donde están alojados, su nivel educativo y edad.

\section{Procedimiento}

El ingreso al centro socioeducativo se realizó luego de solicitar la autorización del directivo de los centros socioeducativos que dependen de la Secretaría de Niñez, Adolescencia y Familia (Senaf) Córdoba, Argentina. El estudio se efectuó en dos etapas, en un primer momento se llevó a cabo un acercamiento a la institución con el fin de lograr una mayor confianza con los jóvenes institucionalizados y, en una segunda etapa, se administraron los instrumentos.

Los cuestionarios se manejaron de manera colectiva con una duración de 50 minutos aproximadamente, dentro del 
horario regular de clases, en coordinación con los directivos de la escuela y los profesores de cada curso; solicitando la colaboración de cada alumno de manera completamente voluntaria y anónima, con el fin de resguardar su identidad. El estudio cumplió con los valores éticos requeridos en la investigación con seres humanos (consentimiento informado y derecho a la información, protección de datos y confidencialidad, gratuidad, no discriminación y posibilidad de abandonar el estudio en cualquiera de sus fases), asimismo se aclaró que los resultados obtenidos serían utilizados únicamente con fines académicos.

Al concluir el estudio se realizó una devolución de los resultados obtenidos a la institución en una reunión de cierre con los docentes y directivos en donde, además, se ofrecieron líneas de intervención para la problemática estudiada.

\section{Instrumentos}

Los instrumentos administrados para lograr los objetivos propuestos fueron:

Escala de adaptación social (Martorell \& González, 1992). Estima el nivel de conducta prosocial en los adolescentes, compuesta por el Cuestionario de Conducta Prosocial (CC-P) y el Cuestionario de Conducta Antisocial (CC-A); fue adaptada en Argentina por Casullo (1998). El primer cuestionario integra un total de 58 ítems con cuatro alternativas de respuestas (Nunca $=1$, Algunas veces $=2$, Muchas veces $=3$, Siempre $=4)$ y con una resolución de cuatro factores: empatía, respeto, sociabilidad, liderazgo. El Cuestionario de Conducta Antisocial, por su parte, se compone de un total de 36 ítems con cuatro alternativas de respuestas (Nunca
$=1$, Algunas veces $=2$, Muchas veces $=$ 3 , Siempre $=4$ ) y con una resolución de tres factores: aislamiento, agresividad y retraimiento/ansiedad. Los ítems indirectos de ambas escalas se evalúan a la inversa. Para su corrección se suman las respuestas correspondientes a cada uno de los factores, obteniendo un puntaje para cada factor.

En lo relativo a la confiabilidad de los instrumentos, se destaca que para la consistencia interna de las escalas el valor mínimo obtenido es ,76 y el máximo ,82. Para la estabilidad temporal que media en un lapso de tres meses el valor mínimo obtenido fue de ,65 y el máximo fue de ,72. Los índices de confiabilidad obtenidos en el presente estudio para la escala de conducta prosocial fueron de $\alpha=, 86$ para el factor empatía; $\alpha=, 36$ para el factor liderazgo; $\alpha=$ , 52 para el factor respeto; y para el factor sociabilidad $\alpha=, 60$. Para la escala de conducta antisocial fue de $\alpha=, 58$ para el factor ansiedad/retraimiento; $\alpha=$ ,69 para el factor aislamiento y para el factor agresividad el valor fue de $\alpha=$ ,66.

Cuestionario de Clima Prosocial Escolar (CCPE) (Roche Olivar, 2004). Mide el clima prosocial percibido por los adolescentes en el contexto escolar. Está compuesto por una estructura de tipo Likert con cinco opciones de respuesta que van desde Muy raramente hasta Casi siempre. Se plantean situaciones y cada estudiante deberá responder de acuerdo con la frecuencia con que considera que se dan dichas situaciones en el colectivo del contexto escolar (aula). Para este cuestionario los valores de fiabilidad obtenidos son para la fase pretest $(\alpha=, 85)$ y para la postest $(\alpha=, 84)$ en una muestra de 178 alumnos de una escuela secundaria en Espa- 
ña (Romersi et al., 2011). El cuestionario no cuenta con estudios de validez y confiabilidad en Argentina.

\section{Cuestionario Prosocial Escolar} (Roche Olivar, 2004). Permite conocer la percepción de los adolescentes respecto de las conductas prosociales de sus compañeros en el contexto escolar. Consta de 10 ítems que corresponden a 10 categorías teóricas de la conducta prosocial (ayuda física, servicio físico, dar, ayuda verbal, consuelo verbal, confirmación y valorización positiva del otro, escucha profunda, empatía, solidaridad, presencia positiva y unidad). Las opciones de respuesta se presentan en una escala tipo Likert con cinco posibilidades que van desde Muy raramente hasta Casi siempre. El cuestionario es de heteroevaluación y se aplica con la finalidad de determinar las veces que se observan conductas prosociales en los compañeros. Para este cuestionario se obtienen valores de fiabilidad para la fase pretest $(\alpha=, 94)$ como en la de postest $(\alpha=, 94)$ en una muestra de 178 alumnos que asisten a la escuela secundaria en España (Romersi et al., 2011).
El cuestionario no cuenta con estudios de validez y confiabilidad en Argentina.

\section{Resultados}

\section{Estudio 1. Características sociodemo-} gráficas de la población

En la tabla 1 es posible observar que un $32,6 \%$ de jóvenes son menores de 16 años, a pesar de que la ley señale que un menor de 16 años no es punible. Respecto de la evaluación de las condiciones familiares, se tuvo en cuenta si los jóvenes vivían con su familia nuclear (madre, padre y hermanos), o si tenían a sus padres separados, vivían con uno de ellos, con sus parejas o con otros familiares; los resultados muestran que el $64,5 \%$ de los jóvenes tiene su familia disgregada, la cantidad de hermanos es un factor importante, resultando una media de 5,41 hermanos por joven, con una $D E=2,97$. Además, se contempló el nivel educativo de la madre y el padre; los resultados muestran que en ambos progenitores el mayor porcentaje ha concluido los estudios de nivel secundario.

Tabla 1

Variables sociodemográficas

\begin{tabular}{lcc}
\hline & $\boldsymbol{f}$ & $\boldsymbol{\%}$ \\
\hline Edad & & \\
Menores de 16 años & 28 & $32,6 \%$ \\
Mayores de 16 años & 58 & $67,4 \%$ \\
Tipos de familia & & \\
Familia nuclear & 27 & $35,5 \%$ \\
Otras tipologías de familiares & 49 & $64,5 \%$ \\
\hline
\end{tabular}

La distribución de alojamiento está divida en cuatro institutos en los que se agrupa a los jóvenes en relación con el delito co- metido, aunque esta delimitación no es taxativa, en la tabla 2 se presenta la capacidad de alojamiento de cada instituto. 
Tabla2

Distribución de jóvenes por alojamiento

\begin{tabular}{lccc} 
Institutos & Edad & Tipo de delito & $\begin{array}{c}\text { Capacidad de } \\
\text { alojamiento }\end{array}$ \\
\hline Módulo A & 17 y 21 años & Delitos graves (homicidios) & 20 camas \\
Módulo B & 15 y 19 años & $\begin{array}{c}\text { Conductas de robo, en ocasio- } \\
\text { nes con homicidio }\end{array}$ & 148 camas \\
Módulo C & 16 y 19 años & Delitos de tipo sexual & 40 camas \\
Módulo D & Menores de 16 años & Delitos de todo tipo & 50 camas \\
\hline
\end{tabular}

Estudio 2. Análisis del nivel de adaptación social en los jóvenes escolarizados

Para estimar el nivel de adaptación social de los jóvenes se dividió al grupo de acuerdo con los puntajes brutos obtenidos por cada sujeto en cada uno de los factores que componen el Cuestionario de Conducta Prosocial y Antisocial. Posteriormente se interpretaron los puntajes utilizando como referencia el baremo elaborado por Casullo (1998) para jóvenes argentinos, presentado en la tabla 3.

Tanto para la conducta prosocial como antisocial se procedió a realizar el análisis descriptivo (media y desviación estándar) de los factores que componen ambos cuestionarios. En segunda instancia se procedió a agrupar a los jóvenes de acuerdo con el nivel alcanzado en cada uno de los factores que componen la conducta prosocial y antisocial.

Como se muestra en la tabla 4 , los resultados señalan que el mayor porcentaje de los jóvenes se encuentra ubicado en el grupo que presenta un nivel bajo de empatía $(47,7 \%)$ y respeto $(41,9 \%)$; así como el $(41,9 \%)$ y el $(91,9 \%)$ se ubica en niveles altos de liderazgo y sociabilidad respectivamente.
Tabla 3

Baremos para la escala de adaptación social

\begin{tabular}{llc}
\hline & Nivel & Puntuación \\
\hline $\begin{array}{l}\text { Conducta } \\
\text { prosocial } \\
\text { Empatía }\end{array}$ & & \\
& Bajo & $0-47$ \\
& Medio & $48-53$ \\
& Alto & Más de 53 \\
Liderazgo & Bajo & $0-24$ \\
& Medio & $24-28$ \\
& Alto & Más de 28 \\
Respeto & Bajo & $0-41$ \\
& Medio & $42-45$ \\
& Alto & Más de 45 \\
Sociabilidad & Bajo & $0-30$ \\
& Medio & $31-33$ \\
& Alto & Más de 33 \\
Conducta & & \\
antisocial & & \\
Agresividad & Bajo & $0-23$ \\
& Medio & $24-27$ \\
& Alto & Más de 27 \\
Aislamiento & Bajo & $0-16$ \\
& Medio & $17-22$ \\
& Alto & Más de 22 \\
& & \\
Retraimiento/ & Bajo & $0-15$ \\
ansiedad & Medio & $15-17$ \\
& Alto & Más de 17 \\
\hline & &
\end{tabular}


Tabla 4

Descriptivos y nivel alcanzado por los alumnos en conducta prosocial y antisocial

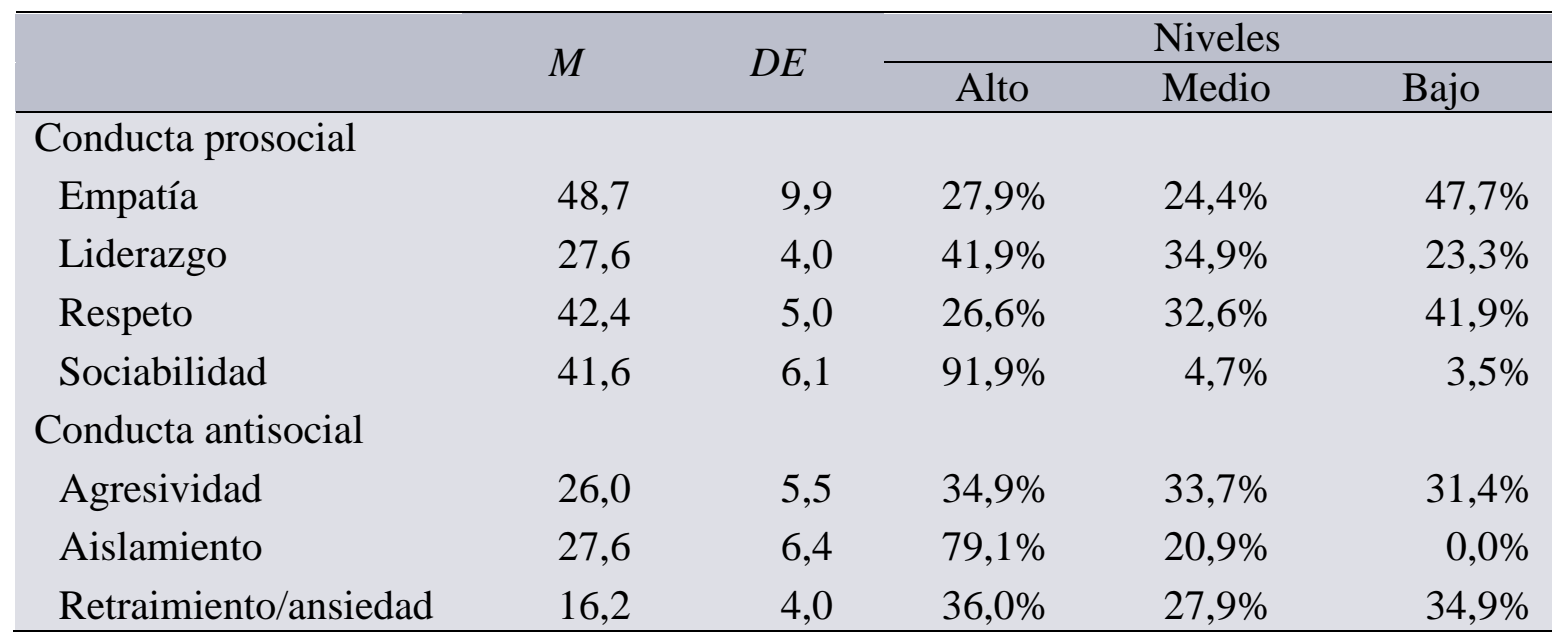

En relación con la conducta antisocial se observa que si bien en el factor agresividad y retraimiento la mayor cantidad de jóvenes se encuentra en un nivel alto $(34,9 \%$ y $36,0 \%$ respectivamente), la distribución de la población para ambos factores, en los diferentes niveles resulta homogénea.

Finalmente un $79,1 \%$ de los jóvenes presenta un nivel alto en el factor aislamiento.
Estudio 3. Análisis descriptivo del clima prosocial escolar percibido por los estudiantes y percepción de conductas prosociales en sus pares

A continuación se procedió con un análisis descriptivo del clima prosocial escolar percibido y la percepción de los alumnos acerca de la presencia de conductas prosociales de sus pares en el contexto escolar (tabla 5). Asimismo, para efectuar un análisis más pormenorizado se evaluó el porcentaje de respuesta para cada una de las conductas contempladas (tabla 6 y 7).

Tabla 5

Estadísticos descriptivos para el cuestionario de clima prosocial escolar

\begin{tabular}{lcccc}
\hline \multicolumn{1}{c}{ Variables } & $M$ & $D E$ & Mínimo & Máximo \\
\hline Clima prosocial escolar percibido & 36,23 & 7,42 & 18 & 50 \\
Conducta prosocial escolar en sus pares & 31,06 & 7,25 & 14 & 48 \\
\hline
\end{tabular}

Estudio 4. Relación entre el clima prosocial escolar y la adaptación social

Con el objetivo de explorar la asociación entre las variables en estudio se calculó el coeficiente de correlación de
Pearson. Como se muestra en la tabla 8, en la mayoría de las relaciones estudiadas las correlaciones son estadísticamente significativas y los coeficientes encontrados se encuentran en el rango moderado. 
Tabla 6

Percepción de los jóvenes sobre el clima prosocial escolar

\begin{tabular}{lrcccc}
\hline Factores & Nunca & Alguna vez & Varias veces & Muy seguido & Siempre \\
\hline Ayuda & $16,7 \%$ & $20,2 \%$ & $11,9 \%$ & $11,9 \%$ & $39,3 \%$ \\
Servicio & $7,1 \%$ & $20,2 \%$ & $17,9 \%$ & $19,0 \%$ & $35,7 \%$ \\
Dar & $7,1 \%$ & $23,8 \%$ & $15,5 \%$ & $21,4 \%$ & $32,1 \%$ \\
Ayuda verbal & $2,4 \%$ & $27,4 \%$ & $17,9 \%$ & $20,2 \%$ & $32,1 \%$ \\
Consuelo verbal & $6,0 \%$ & $15,5 \%$ & $10,7 \%$ & $19,0 \%$ & $48,8 \%$ \\
Confirmación & $7,1 \%$ & $25,0 \%$ & $19,0 \%$ & $22,6 \%$ & $26,2 \%$ \\
Escucha & $3,6 \%$ & $19,0 \%$ & $20,2 \%$ & $14,3 \%$ & $42,9 \%$ \\
Empatía & $10,7 \%$ & $19,0 \%$ & $10,7 \%$ & $22,6 \%$ & $36,9 \%$ \\
Solidaridad & $4,8 \%$ & $15,5 \%$ & $20,2 \%$ & $17,9 \%$ & $41,7 \%$ \\
Presencia positiva & $4,8 \%$ & $16,7 \%$ & $7,1 \%$ & $17,9 \%$ & $53,6 \%$ \\
\hline
\end{tabular}

Tabla 7

Percepción de los jóvenes sobre las conductas prosociales de sus pares en el contexto escolar

\begin{tabular}{lrcccr}
\hline Factores & Nunca & Alguna vez & Varias veces & Muy seguido & Siempre \\
\hline Ayuda & $12,1 \%$ & $25,8 \%$ & $30,3 \%$ & $22,7 \%$ & $9,1 \%$ \\
Servicio & $22,7 \%$ & $16,7 \%$ & $28,8 \%$ & $21,2 \%$ & $10,6 \%$ \\
Dar & $4,5 \%$ & $18,2 \%$ & $28,8 \%$ & $25,8 \%$ & $22,7 \%$ \\
Ayuda verbal & $13,6 \%$ & $28,8 \%$ & $33,3 \%$ & $21,2 \%$ & $3 \%$ \\
Consuelo verbal & $6,1 \%$ & $22,7 \%$ & $28,8 \%$ & $24,2 \%$ & $18,2 \%$ \\
Confirmación & $9,1 \%$ & $22,7 \%$ & $33,3 \%$ & $24,2 \%$ & $10,6 \%$ \\
Escucha & $10,6 \%$ & $28,8 \%$ & $28,8 \%$ & $18,2 \%$ & $13,6 \%$ \\
Empatía & $4,6 \%$ & $21,5 \%$ & $33,8 \%$ & $18,5 \%$ & $21,5 \%$ \\
Solidaridad & $9,2 \%$ & $21,5 \%$ & $23,1 \%$ & $29,2 \%$ & $16,9 \%$ \\
Presencia positiva & $10,8 \%$ & $16,9 \%$ & $21,5 \%$ & $21,5 \%$ & $29,2 \%$ \\
\hline
\end{tabular}

Se constatan las correlaciones positivas previstas teóricamente entre los puntajes de la conducta prosocial que presentan los sujetos (sociabilidad, liderazgo, empatía, respeto) con el clima prosocial escolar percibido (ayuda física, servicio físico, dar, ayuda verbal, consuelo verbal, confirmación y valorización positiva del otro, escucha profunda, empatía, solidaridad, presencia positiva y unidad) $(r=, 511 ; p<$ $, 01)$. Esto es, a mayor presencia de conducta prosocial en los sujetos, mayor per- cepción en relación al clima prosocial en el ámbito áulico y viceversa. Por otro lado, se observa que la percepción del clima prosocial escolar por parte de los jóvenes correlaciona positivamente con la consideración que ellos tienen respecto de la presencia de conductas prosociales en cada uno de sus compañeros (ayuda física, servicio físico, dar, ayuda verbal, consuelo verbal, confirmación y valorización positiva del otro, escucha profunda, empatía, solidaridad, presencia positiva y 
unidad) $(r=, 526 ; p<, 01)$. Esto pone en evidencia que la evaluación que realizan los jóvenes de un clima escolar prosocial contribuye a fortalecer la mirada positiva que tienen de sus pares y viceversa. Por último, en concordancia con la teoría, se observa una correlación baja y negativa entre clima prosocial escolar y conducta antisocial $(r=-, 106)$, que pone de manifiesto que el incremento de los comportamientos antisociales en los jóvenes puede afectar el clima prosocial escolar que se establece en el aula; sin embargo, al no ser una correlación significativa, demuestra que el clima prosocial escolar va más allá de la ausencia de conductas antisociales.

Tabla 8

Correlación entre adaptación social, clima prosocial escolar y percepción de conductas prosociales

\begin{tabular}{lcccc}
\hline & CPE & PCPP & CA & CP \\
\hline $\begin{array}{l}\text { Clima prosocial escolar (CPE) } \\
\begin{array}{l}\text { Percepción de conductas prosociales en sus pares } \\
\text { (PCPP) }\end{array}\end{array}$ & 1 &, $526^{* *}$ &,- 106 &, $511^{* *}$ \\
$\begin{array}{l}\text { Conducta antisocial (CA) } \\
\text { Conducta prosocial (CP) }\end{array}$ & &,- 150 &, $292^{*}$ \\
\hline
\end{tabular}

Nota: $* p<.05 * * p<.01$.

\section{Discusión y conclusiones}

A partir del presente trabajo se buscó analizar la adaptación social presente en los jóvenes infractores de ley en el contexto escolar y que se encuentran alojados en un centro socioeducativo cerrado. Para ello, se procedió a describir el nivel de adaptación social (conducta prosocial y conducta antisocial) de los jóvenes. Los resultados obtenidos muestran que, en conducta prosocial, los jóvenes encuestados se encuentran en un nivel bajo para factores como empatía y respeto; sin embargo, en lo que respecta a liderazgo y sociabilidad, los niveles alcanzados para la mayoría son altos. Estos datos son relevantes, ya que de acuerdo con lo expresado por Martorell et al. (2011b) en la adolescencia la empatía, el respeto por los demás, las relaciones sociales y el liderazgo se encuentran en evolución.

Consecuentemente con lo expresado en diversas investigaciones para este tipo de población (Graña Gómez \& Rodríguez Biezma, 2010; Mestre Escrivá, Samper García, \& Frías Navarro, 2002), los puntajes obtenidos en este estudio para empatía son bajos. Siguiendo a Martorell et al. (2011b) se entiende la empatía como la capacidad de ponerse en el lugar del otro y la búsqueda de alivio a su malestar, la cual se encuentra relacionada directamente con la conducta prosocial, pudiendo actuar como un modulador del comportamiento del joven. Tal como se observa en este estudio y en concordancia con Farrington (1998), la falta de empatía es considerada un factor de riesgo importante para el desarrollo de la conducta criminal. De la misma manera, Martorell et al. (2011a) destacan que la activación emocional empática tiene una influencia en el tipo de respuesta que dará un sujeto ante las reacciones emocionales de los demás; por lo que se considera primordial promover el desarrollo de la empatía en los jóvenes infractores, ya que su fortalecimiento contribuiría a 
un mayor control de la impulsividad, así como de la agresividad.

Conjuntamente, diversos estudios coinciden al sostener que los jóvenes que muestran conductas antisociales suelen presentar déficits en la capacidad para tratar a los demás con respeto y asertividad (Farrington, 1998; Loeber, Farrington, \& Petechuk, 2003). En coincidencia con lo mencionado, los resultados obtenidos para este estudio muestran que un $41,9 \%$ de los jóvenes presenta un nivel bajo de respeto, el cual es entendido como la capacidad de la persona para tratar a los demás con respeto y asertividad (Martorell et al., 2011b).

En tanto, los puntajes obtenidos para el factor sociabilidad demuestran que un 92\% de los jóvenes está en condiciones de llevar a cabo relaciones sociales efectivas (Martorell et al., 2011b). Sin embargo, esto no resulta llamativo, dado que los criminólogos han caracterizado a la delincuencia juvenil como una delincuencia de grupos (Garrido, Stangeland, \& Redondo Illescas, 2006). Asimismo, los resultados encontrados para el factor liderazgo muestran que el mayor porcentaje de los jóvenes obtiene un nivel alto, lo cual indica que en su mayoría estos poseen la capacidad de organizar y dirigir actividades en grupo (Martorell et al., 2011b). Cabe resaltar aquí que las investigaciones en relación con el estudio y la cultura del liderazgo indican que no siempre se pueden encontrar modelos en donde se promueva el bien común. En este sentido se destaca que un buen líder debería ser, por naturaleza, prosocial, ya que el liderazgo prosocial se expande más allá de lo que se ha estudiado como características del líder y se focaliza en un liderazgo que tiende servir al bien común (Cirera, 2010). Restaría indagar en este estudio qué tipo de liderazgo prevalece.
En lo que refiere a la conducta antisocial, los resultados muestran que el mayor porcentaje de jóvenes presenta un alto nivel de agresividad, entendida como toda conducta que implique agresividad verbal o física en relación con los demás (Martorell et al., 2011a). Sin embargo, siguiendo la línea de algunas investigaciones, se concluye que los jóvenes delincuentes violentos y crónicos suponen una fracción del total de jóvenes, pero suelen ser responsables de la mayoría de los arrestos, ya que en realidad solo un pequeño porcentaje desarrolla una carrera delictiva adulta. De lo anterior se desprende que no todos los delincuentes juveniles terminan desarrollando una carrera delictiva en la adultez (Garrido et al., 2006), por lo que resulta de suma importancia apuntar al trabajo con aquellos jóvenes que presentan un alto nivel de agresión.

En relación con el aislamiento, se observó que un mayor porcentaje de jóvenes presenta un nivel alto, lo cual demostraría que presentan una necesidad no adaptativa de estar solos, huyendo de situaciones que impliquen relacionarse con los demás (Martorell et al., 2011a). Este resultado, a su vez, puede estar vinculado con los efectos que la prisionalización suele conllevar en los internos, entre los que se encuentran sentimientos de aislamiento y ausencia de afectos y relaciones con otros (Altamirano Argudo, 2013; Echeverry Vera, 2010; Fridman et al., 2011; Hartwell, Fisher, \& Davis, 2010; Herrera Enríquez \& Expósito Jiménez, 2010; Segovia \& Valle, 2013).

Finalmente, en el factor retraimiento, aunque la población se presente repartida de manera relativamente homogénea en niveles bajo, medio y alto, los resultandos demuestran que un $36,5 \%$ de los jóvenes infractores se encuentra en el nivel más alto, concordando con lo señalado por 
Martorell et al. (2011a) en esta franja etaria: los jóvenes pueden presentar, en determinadas situaciones, dificultades para compartir espacios con el resto, poniendo en evidencia la dificultad que presentan para relacionarse con otros.

Atendiendo a los resultados obtenidos para evaluar la adaptación social en los jóvenes del centro socioeducativo, se puede resumir que los indicadores que refieren a la conducta antisocial se presentan en mayor medida que los indicadores vinculados con los comportamientos prosociales en los jóvenes, como cabe de esperar en esta población. En relación con el estudio de la prosocialidad en el contexto escolar, teniendo en cuenta que no existen baremos adaptados en Argentina, los resultados muestran que cuando se evalúa la percepción del clima prosocial escolar, el mayor porcentaje de jóvenes señala que las conductas se presentan siempre en los 10 factores que lo componen. Asimismo, al evaluar la percepción de la presencia de conductas prosociales en los pares, el mayor porcentaje de jóvenes señala que todos los factores se presentan varias veces, exceptuando solidaridad $\mathrm{y}$ presencia positiva, los cuales se dan muy seguido $(29,2 \%)$ y siempre $(29,2 \%)$, respectivamente. En relación a esto, Romersi et al. (2011) señalan que los jóvenes suelen hacer una valoración conjunta de la prosocialidad individual y colectiva.

A su vez, el estudio de correlación demostró que existe una relación directa y significativa entre la conducta prosocial a nivel personal y el clima prosocial desarrollado en el aula. Este resultado puede llegar a valorarse positivamente, si se tiene en cuenta que la base de la conducta delictiva está centrada en el déficit de competencias psicosociales y que estas pueden ser aprendidas (Redondo Illescas et al., 2011). De la misma manera, Ro- mersi et al. (2011) señalan que las dimensiones individual y colectiva de la prosocialidad son interdependientes. En este sentido es posible pensar el contexto escolar, y específicamente el aula, como un espacio propicio para la optimización de conductas prosociales (Roche Olivar, 2011). La aplicación de un programa de incremento prosocial ha demostrado resultados positivos, mejorando la percepción de la calidad de las relaciones establecidas en el contexto escolar (Romersi et al., 2011).

A su vez, se observa una correlación negativa entre conducta antisocial y percepción del clima prosocial escolar. En relación con esto, Cava, Musitu y Murghi (2007) señalan que los comportamientos antisociales suelen desarrollarse en el contexto escolar, afectando las relaciones sociales que se establecen en el aula. Afirman que la percepción que los jóvenes tienen acerca del clima escolar puede estar relacionada con su ajuste social en el aula, pudiendo ser consecuencia de su rendimiento académico, su participación en clases, autoestima, sentimientos de soledad y adaptación social.

Además, cabe resaltar la ausencia de relación significativa entre conductas antisociales y conductas prosociales percibidas en el contexto escolar. Los datos indican que una baja conducta antisocial no se asocia a una mayor conducta prosocial en el aula. Es decir que, para aumentar la prosocialidad escolar, no bastaría simplemente con disminuir los comportamientos antisociales, sino que es necesario un entrenamiento enfocado en el incremento de conductas prosociales en jóvenes infractores. Por lo tanto, el presente estudio permite concluir que la deficiencia en conductas prosociales es un factor de riesgo que se relaciona con la delincuencia juvenil, al ser un fenómeno 
de desadaptación social, lo cual es consecuente con la teoría y los hallazgos empíricos, y su ausencia se manifiesta en el contexto escolar.

No obstante, se trata de una primera aproximación en este campo en muestras de jóvenes argentinos en conflicto con la ley penal y se requieren nuevas investigaciones que profundicen en el análisis tanto de la conducta prosocial, como de otras variables psicológicas, relacionadas con la delincuencia juvenil.

En cuanto a las limitaciones presentadas por el estudio es necesario señalar que los cuestionarios de clima prosocial escolar, en sus versiones para evaluar el clima del aula y las conductas de sus compañeros, no cuentan con estudios de validez y confiabilidad realizados en Argentina; no obstante, los valores de fiabilidad obtenidos para esta investigación son altos, (Cuestionario de Clima Prosocial Escolar $\alpha=, 74$ y Cuestionario Prosocial Escolar (compañeros) $\alpha=, 81$, lo cual constituye un avance en su validación para el uso con población infractora juvenil).
En esta vía un desafío a futuro sería desarrollar intervenciones que resulten innovadoras, a través de la generación de espacios que posibiliten a los jóvenes reflexionar acerca de la conducta antisocial y ensayar nuevas conductas prosociales, que les permitan elaborar alternativas de comportamiento útiles para reintegrarse con mayor éxito en la sociedad. El docente, a través de un adecuado entrenamiento, puede servir de modelo y de agente modelador de conductas prosociales con el fin de reforzar las mismas en los jóvenes.

Igualmente es importante pensar los centros socioeducativos para jóvenes delincuentes como espacios propicios para el desarrollo y promoción de habilidades psicosociales que se encuentran de manera deficitaria o que no están presentes. El modelo de Roche Olivar (2004) antes citado, puede ser de gran utilidad en tanto posibilita el trabajo de conductas prosociales de manera transversal en el aula, ámbito destacado como valioso para estos jóvenes.

\section{Referencias}

Akers, R. L. (2009). Social learning and social structure: A general theory of crime and deviance. New Jersey: Transaction Publishers.

Akers, R. L. \& Burgess, R. L. (1966). A differential association reinforcement theory of criminal behavior. Social Problems, 14(2), 128-147.

http://dx.doi.org/10.1525/sp.1966.14.2.03a00020

Alarcón Bañares, P., Pérez-Luco, R., Salvo, S., Roa, G., Jaramillo, K., \& Sanhueza, C. (2010). Validación del cuestionario de auto-reporte de comportamiento antisocial en adolescentes: CACSA1. Paidéia, 29(47), 291-302. http://dx.doi.org/10.1590/S0103-863X2010000300002

Altamirano Argudo, Z. (2013). El bienestar psicológico en prisión: antecedentes y consecuencias. Recuperado de http://hdl.handle.net/10486/13008

Bandura, A. (1977). Teoría del aprendizaje social. Madrid: Espasa-Calpe. 
Barrón, M. \& Carbonetti, M. (2006). La escuela como factor de inclusión en la adolescencia. En M. Barrón (Comp.), Violencia: serie adolescencia, educación y salud 2 (pp. 3142). Córdoba: Brujas.

Casullo, M. (1998). Adolescentes en riesgo. Buenos Aires: Paidós.

Cava, M. J., Musitu, G., \& Murgui, S. (2007). Individual and social risk factors related to overt victimization in a sample of Spanish adolescents. Psychological Reports, 101(1), 275-290. http://dx.doi.org/10.2466/pr0.101.1.275-290

Cirera Amores, M., Escotorín Soza, P., \& Roche Olivar, R. (2008). Diploma civismo, conductas sociales positivas y socialización. Aplicaciones de la prosocialidad al desarrollo y educación de las actitudes y conductas cívicas. Recuperado de http://is.gd/50WGhp

Cirera, M. (2010). Liderazgo prosocial: hacia un liderazgo participativo y eficiente. En R. Roche Olivar (Comp.), Prosocialidad, nuevos desafios: métodos y pautas para la optimización creativa del entorno (pp. 99-109). Buenos Aires: Ciudad Nueva.

Crabay, M. I. (2007). Adolescencias y juventudes, desafíos actuales. Córdoba, Argentina: Brujas.

D’angelo, L. \& Fernández, D. (2011). Clima, conflictos y violencia en la escuela. Recuperado de http://www.unicef.org/argentina/spanish/clima_conflicto_violencia_escuelas.pdf

Eberly, D. \& Roche Olivar, R. (2002). Aprendizaje-servicio y prosocialidad. Buenos Aires: CLAYSS.

Echeverri Vera, J. A. (2010). La prisionalización, sus efectos psicológicos y su medición. Revista Pensando Psicología, 6(11), 157-166. Recuperado de http://revistas.ucc.edu.co/index.php/pe/article/viewFile/375/378

Farrington, D. P. (1998). Predictors, causes, and correlates of male youth violence. Crime and Justice, 24, 421-475.

http://dx.doi.org/10.1086/449284

Fridman, D., Salgado, V., López, A., Pasín, J., Jorolinsky, K. F. G., \& Guemureman, S. (2011). Entre la ley y la práctica: vida cotidiana en las instituciones cerradas para adolescentes. En A. Daroqui \& S. Guemureman (Coord.), Sistema penal y derechos humanos. IX Jornada de Sociología Pre-Alas Recife: Capitalismo del siglo XXI, crisis y reconfiguraciones. Simposio llevado a cabo en Facultad de Ciencias Sociales, Universidad de Buenos Aires, Buenos Aires, Argentina.

Garrido, V., Stangeland, P., \& Redondo Illescas, S. (2006). Principios de criminología. Valencia: Tirant lo Blanch.

González, R., Casullo, M., Martorell, C., \& Calvo, A. (1998). Evaluación de los comportamientos sociales. Aportaciones de un estudio comparativo. En M. Casullo (Comp.), Adolescentes en riesgo: identificación y orientación psicológica (pp. 127-144). Buenos Aires, Argentina. 
Graña Gómez, J. L. \& Rodríguez Biezma, M. J. (2010). Programa central de tratamiento educativo y terapéutico para menores infractores. Agencia de la comunidad de Madrid para la reeducación y reinserción del menor infractor. Recuperado de http://www.observatoriodelainfanciadeasturias.es/documentos/f07022012130358.pdf

Hartwell, S., Fisher, W., \& Davis, M. (2010). Emerging adults with psychiatric disabilities Involved with the criminal justice system. International Journal of Offender Therapy and Comparative Criminology, 54(5), 756-768.

http://dx.doi.org/10.1177/0306624x09338018

Herrera Enríquez, M. \& Expósito Jiménez, F. (2010). Una vida entre rejas: aspectos psicosociales de la encarcelación y diferencias de género. Psychosocial Intervention, 19(3), 235-141.

http://dx.doi.org/10.5093/in2010v19n3a4

Kornblit, A., Adaszko, D., \& Di Leo, P. (2008). Clima social escolar y violencia: un vínculo explicativo posible. En A. Kornblit (Coord.), Violencia escolar y climas sociales (pp. 59-79). Buenos Aires: Biblós.

Loeber, R., Farrington, D., \& Petechuk, D. (2003). Child delinquency: Early intervention and prevention. Recuperado de

https://www.ncjrs.gov/pdffiles1/ojjdp/186162.pdf

Lorenzo Moledo, M., Aroca Montolío, C., \& Alba Robles, J. (2013). La pedagogía penitenciaria en España: luces y sombras. Revista de Educación, 360, 119-139.

http://10.4438/1988-592X-RE-2013-360-223

Maganto, C. (1994). Influencia de la familia y la escuela en la socialización y la conducta prosocial. En M. Garaigordobil \& C. Maganto (Eds.), Socialización y conducta prosocial en la infancia y la adolescencia (pp. 37-71). España: Argitalpen zerbitzua

Martorell, C. \& González, B. R. (1992). Cuestionarios de conducta prosocial y antisocial. En R. González, M. Casullo, C. Martorell, \& A. Calvo (Eds.), Evaluación de los comportamientos sociales, aportaciones de un estudio comparativo (pp. 127-144). Madrid: Morata

Martorell, C., González, R., Ordoñez, A., \& Gómez, O. (2011a). Estudio confirmatorio del Cuestionario de Conducta Antisocial (CCA) y su relación con variables de personalidad y conducta antisocial. Revista Iberoamericana de Diagnóstico y Evaluación Psicológica, 31(1), 97-114. Recuperado de http://www.aidep.org/03_ridep/R31/R31\%20art5.pdf

Martorell, C., González, R., Ordoñez, A., \& Gómez, O. (2011b). Estudio confirmatorio del Cuestionario de Conducta Prosocial (CCP) y su relación con variables de personalidad y socialización. Revista Iberoamericana de Diagnóstico y Evaluación Psicológica, 32(2), 35-40. Recuperado de http://www.aidep.org/03_ridep/R32/r32art2.pdf

Mestre Escrivá, M., Samper García, P., \& Frías Navarro, M. (2002). Procesos cognitivos y emocionales predictores de la conducta prosocial y agresiva: la empatía como factor modulador. Psicothema, 14(2), 227-232. Recuperado de http://www.psicothema.com/psicothema.asp?id=713 
Moffit, T. E. (1993). Adolescence-limited and life-course-persistent antisocial behavior: A developmental taxonomy. Psychological Review, 100(4), 674-701.

http://dx.doi.org/10.1037//0033-295x.100.4.674

Moffit, T. E., Caspi, A., Harrington, H., \& Milne, B. J. (2002). Males on the life-coursepersistent and adolescence-limited antisocial pathways: Follow-up at age 26 years. Development and Psychopathology, 14(1), 179-207.

http://dx.doi.org/10.1017/s0954579402001104

Moffitt, T. E. (2005). The new look of behavioral genetics in developmental psychopathology: Gene-environment interplay in antisocial behaviors. Psychological bulletin, 131(4), 533-554.

http://dx.doi.org/10.1037/0033-2909.131.4.533

Redondo Illescas, S., Martínez Catena, A., \& Andrés Pueyo, A. (2011). Factores de éxito asociados a los programas de intervención con menores infractores. Universidad de Barcelona: Ministerio de Sanidad, Política Social e igualdad. Recuperado de http://www.observatoriodelainfancia.msssi.gob.es/productos/pdf/factoresDeExito.pdf

Redondo Illescas, S., Martínez Catena, A., \& Andrés Pueyo, A. (2012). Intervenciones con delincuentes juveniles en el marco de la justicia: investigación y aplicaciones. EduPsykhé, 11(2), 143-169. Recuperado de

http://is.gd/2gdZd5

Roche Olivar, R. (2004). Desarrollo de la inteligencia emocional y social desde los valores y actitudes prosociales en la escuela: guía práctica para la enseñanza y el aprendizaje vital en alumnos del EGB3. Buenos Aires: Ciudad Nueva.

Roche Olivar, R. (2011). Psicología y educación para la prosocialidad. Buenos Aires: Ciudad Nueva.

Romersi, S., Martínez Fernández, J., \& Roche Olivar, R. (2011). Efectos del programa mínimo de incremento prosocial en una muestra de estudiantes de educación secundaria. Anales de Psicología, 27(1), 135-146. Recuperado de http://revistas.um.es/analesps/article/view/113561/107551

Segovia, M. \& Valle, M. (2013). Situación de derechos humanos en instituciones de privación de libertad que alojan a jóvenes menores de 18 años en Córdoba. En L. Bonafé, M. Brocca, J. Burijovich, E. Garbarino Pico, S. Gezmet, L. Luque, ... M. Segovia. (Eds.), Informe provincial mirar tras los muros: situación de los derechos humanos de las personas privadas de libertad en Córdoba. Córdoba: Universidad Nacional de Córdoba. Recuperado de http://is.gd/YXkqoi

Trudel, M., Puentes Neuman, G., \& Ntebutse, J. (2002). Les conceptions contemporaines de l'enfant à risque et la valeur heuristique du construit de résilience en éducation. $\mathrm{Ca}$ nadian Journal of Education, 27, 2-3, 153-173.

http://dx.doi.org/10.2307/1602218

Fecha de recepción: 30 de enero de 2015 Fecha de aceptación: 8 de junio de 2015 